\title{
Single-Virus Force Spectroscopy Discriminates the Intrinsic Role of Two Viral Glycoproteins upon Cell Surface Attachment
}

\author{
Martin Delguste, Grégoire Le Brun, Florian Cotin, Bénédicte Machiels, Laurent Gillet, \\ and David Alsteens*
}

Cite This: Nano Lett. 2021, 21, 847-853

Read Online

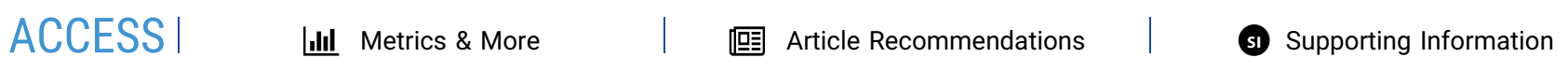
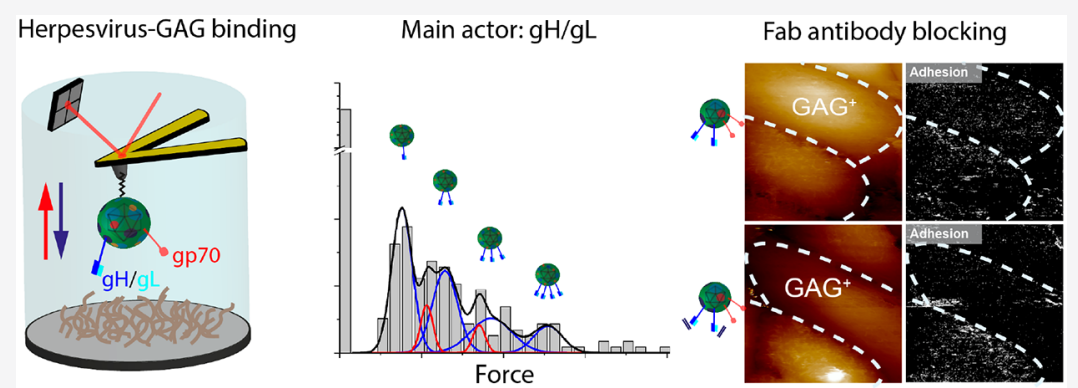

ABSTRACT: Viruses are one of the most efficient pathogenic entities on earth, resulting from millions of years of evolution. Each virus particle carries the minimum number of genes and proteins to ensure their reproduction within host cells, hijacking some host replication machinery. However, the role of some viral proteins is not yet unraveled, with some appearing even redundant. For example, murid herpesvirus 4, the current model for human gammaherpesvirus infection, can bind to cell surface glycosaminoglycans using both glycoproteins gp70 and $\mathrm{gH} / \mathrm{gL}$. Here, using atomic force microscopy, we discriminate their relative contribution during virus binding to cell surface glycosaminoglycans. Single-virus force spectroscopy experiments demonstrate that $\mathrm{gH} / \mathrm{gL}$ is the main actor in glycosaminoglycan binding, engaging more numerous and more stable interactions. We also demonstrated that Fab antibody fragments targeting $\mathrm{gH} / \mathrm{gL}$ or gp70 appear to be a promising treatment to prevent the attachment of virions to cell surfaces.

KEYWORDS: virus-cell interactions, glycoproteins, glycosaminoglycans, atomic force microscopy, single-virus force spectroscopy

T $\mathrm{n}$ order to infect cells, viruses must specifically bind to 1 permissive cell surfaces and find strategies to cross their plasma membrane to replicate within the cells. The initial attachment step consists of the virus particles binding to cellular glycans or proteins and is mediated by the viral surface (glyco)proteins, which define the entry routes and uptake mechanisms exploited by virions. ${ }^{1}$ Depending on the targeted cell type and characteristics, millenary evolution of viruses has led to the development of various uptake mechanisms. These tightly regulated mechanisms assign very specific roles to the viral surface glycoproteins involved in cell attachment. For many viruses, only one glycoprotein mediates cellular binding and membrane fusion (e.g., influenza viruses ${ }^{2}$ ), whereas other viruses devote multiple glycoproteins to this step (e.g., hepatitis $\mathrm{C}$ virus $^{3}$ ). In the latter case, simultaneous or sequential glycoprotein recruitment is often essential to ensure successful infection. ${ }^{4}$ While the identity of the glycoproteins involved in cellular binding is well-described for numerous viral species, their precise role in multistep binding process to cell surfaces is often missing. As the interplay between different glycoproteins is a dynamic process, it is essential to characterize virus binding events at the molecular scale and at high temporal and spatial resolution ${ }^{5-7}$ to get a full picture of this complex multistep attachment processes.

Many viruses exploit sugar moieties such as glycosaminoglycans (GAGs) as a first anchor to cellular surfaces. For example, herpesviruses (HVs) are highly dependent on heparan sulfate for efficient cell binding and infection. ${ }^{8} \mathrm{HVs}$ are ubiquitous pathogens persistently infecting a wide variety of vertebrates. Among them, murid herpesvirus 4 (MuHV-4) is a model of choice to study human gammaherpesvirus pathogenesis. 9 MuHV-4 encodes at least five glycoproteins devoted to cell binding, among which at least three are involved in GAG binding. The heterodimer $\mathrm{gH} / \mathrm{gL}$ and the glycoprotein gp70 directly bind GAGs, whereas the glycoprotein gp150 regulates virus attachment upon GAG-binding. ${ }^{10-12}$ Current knowledge indicates that the two glyco-

Received: November 20, 2020

Revised: December 22, 2020

Published: December 29, 2020 
A
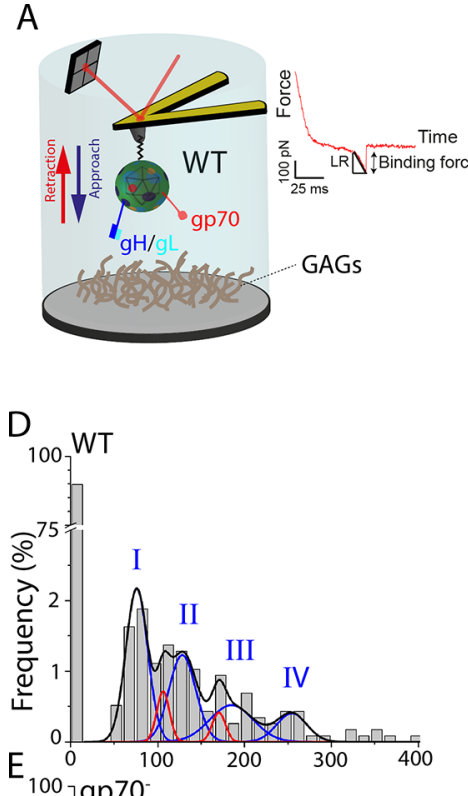

$\mathrm{E}_{100}{ }^{0} \mathrm{gp} 70^{-}$
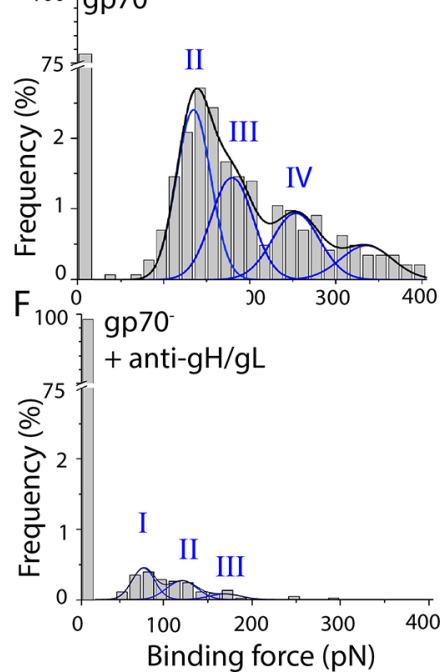

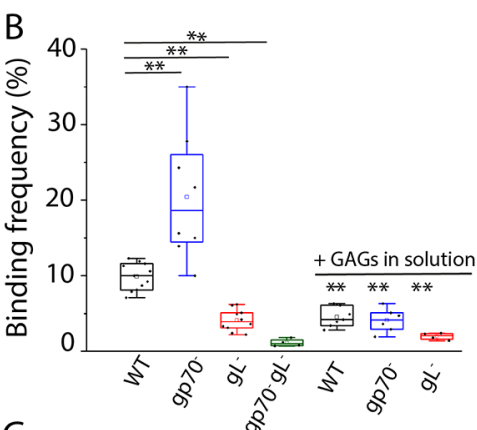

G
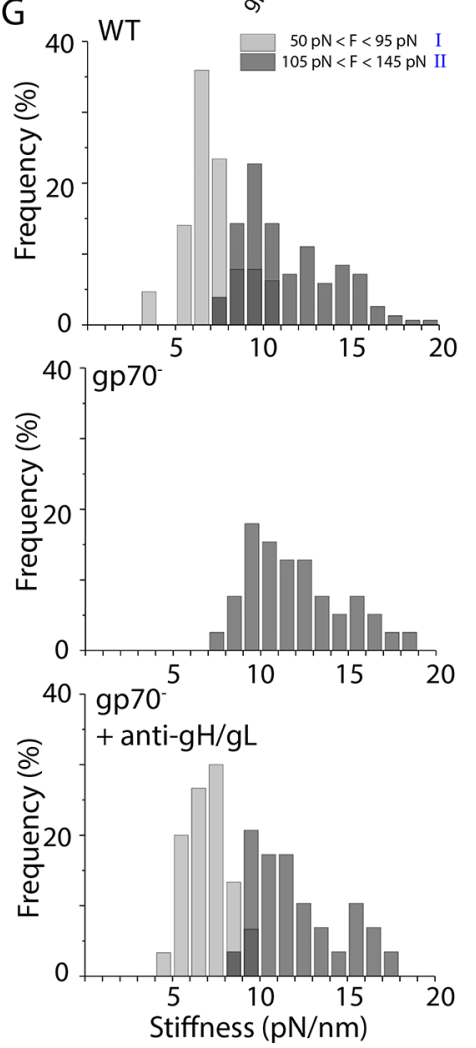

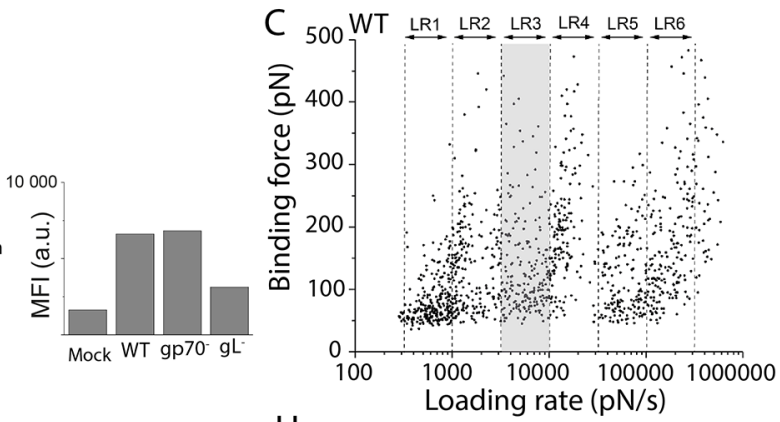

$\mathrm{H}$
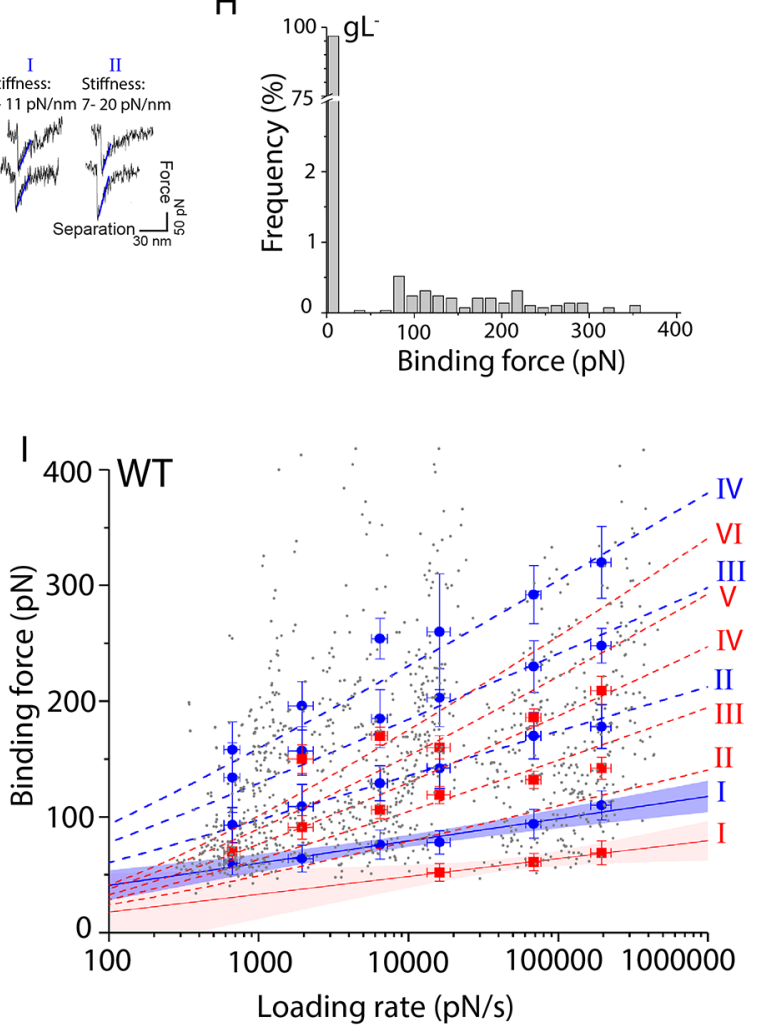

Figure 1. MuHV-4 WT and mutant virions binding to GAG-coated surfaces. (A) Cartoon showing and AFM tip functionalized with an MuHV-4 viral particle probing a GAG-coated model surface. Representative force-time curve showing how the binding force and the loading rate (LR) are extracted. (B) Box plot of WT and mutant binding frequencies, before and after injection of GAGs in solution (** indicates $p$-values $<0.05$ on paired sample $t$ tests). Right: virus binding assays performed on living cells. Mean fluorescence intensity (MFI) of cells is an indicator of the number of attached viral particles. (C) Dynamic force spectroscopy (DFS) plot showing the force required to separate single WT MuHV-4 particles from GAG molecules. Each dot corresponds to a single rupture event $(N=1200$ from four independent experiments). The DFS plot is separated in narrow LR distributions. (D-F) Distribution of binding forces of (D) WT virions $(N=160)$, (E) gp $70^{-}$virions $(N=315)$, and $(\mathrm{F})$ gp70 virions + anti-gH/gL antibodies $(N=40)$ for the third $\mathrm{LR}$ range $(3150-10000 \mathrm{pN} / \mathrm{s})$ (four independent experiments). The histograms are fitted with multigaussian fits. (G) Histograms of bonds stiffness extracted from curves originating from single and double $\mathrm{gH} / \mathrm{gL}$ bonds for WT virions $(N=210)$ and gp $70^{-}$virions in the presence $(N=60)$ and absence $(N=55)$ of anti-gH/gL antibodies (4 independent experiments). Right: force-separation curves originating from single and double bonds from which the stiffness of the bonds probed can be extracted.(H) Binding forces histogram of $\mathrm{gL}^{-}$virions for the third LR range $(N=100,8$ independent experiments). (I) DFS plot reporting the average values of the force distribution for WT MuHV-4-GAG interactions, enabling analysis with the Bell-Evans model (full lines). Dashed lines represent the predicted binding forces for multiple uncorrelated interactions breaking simultaneously, according to the Williams-Evans model. Gray dots represent the force and LR values extracted from each individual FD curve.

proteins $\mathrm{gH} / \mathrm{gL}$ and $\mathrm{gp} 70$ show some redundancy in their GAG-binding properties. Indeed, virus blocking by anti-gH/gL or anti-gp70 antibodies individually does not prevent cell binding, whereas together these antibodies are strong binding inhibitors. ${ }^{12}$ The reason for this redundancy remains unclear, as it seems unlikely from an evolutionary standpoint that a viral species would affect two different glycoproteins to accomplish the same task. Therefore, the exact roles of $\mathrm{gH} / \mathrm{gL}$ and $\mathrm{gp} 70$ upon GAG-binding remain to be clarified.
In this study, we force-probe using atomic force microscopy (AFM) the binding properties of MuHV-4 virions toward cellular GAGs, with the aim of deciphering the putative roles of $\mathrm{gH} / \mathrm{gL}$ and gp70 upon virus attachment. We have recently shown that AFM, and in particular single-virus force spectroscopy, enables the extraction of the binding and kinetic properties of viral glycoproteins interactions with GAGs at the single-virion level. ${ }^{13,14}$ Using this approach, we investigated the multivalent binding mechanism of MuHV-4 particles toward 
GAGs. This let us conclude that this multivalency was tightly regulated by the viral glycoprotein gp150. Here, we discriminate for the first time the distinct roles of two apparently redundant GAG-binding viral glycoproteins. We observe that the virus attachment step is primarily mediated by the establishment of rather stable $\mathrm{gH} / \mathrm{gL}-\mathrm{GAG}$ interactions, whereas the GAG-binding properties of gp70 have only a minor impact upon virus binding and presumably find their importance in another step of the viral cycle. We also evidence that antibody and in particular Fab antibody fragments are valid approaches to prevent virions' early attachment to cellsurface GAGs.

\section{RESULTS}

gL Deletion Strongly Reduces Virus Interaction with GAGs. As we first wanted to evaluate the binding of MuHV-4 virions toward immobilized GAGs, we performed forcedistance curve-based AFM (FD-based AFM) with tips derivatized with single MuHV-4 particles on immobilized GAGs, mimicking cellular surfaces (Figure 1A and Figure S1). Tips were then approached and retracted from GAG surfaces to record force vs time and force vs distance curves. Upon breaking of interactions formed between virions and GAGs, rupture events can be observed on the retraction curves, enabling us to quantify the force required to break the interactions (i.e., the binding force) (Figure 1A). We used AFM tips functionalized with four different viral particles: wildtype MuHV-4 virions (WT) and MuHV-4 virions lacking either the glycoprotein $\mathrm{gL}$, which is essential to allow $\mathrm{gH}$ to adopt its GAG-binding conformation $\left(\mathrm{gL}^{-}\right)$, the glycoprotein gp70 $\left(\mathrm{gp} 70^{-}\right)$, or both $\left(\mathrm{gp} 70^{-} \mathrm{gL}^{-}\right)$. From the FD curves recorded, we extracted the ones showing the characteristic signature of a specific rupture event. To validate the specificity of these rupture events, we fitted the adhesion peak with the wormlike chain model to confirm the typical pattern of polymer elongation (Figure S2) and performed two independent controls using a PEG-coated tip and the in situ injection of GAGs in solution (Figure $1 \mathrm{~B}$ and Figure S2). We then compared the binding frequencies (i.e., the number of curves showing specific rupture events compared to the total number of curves recorded) of the different viral particles. WT MuHV-4 virions displayed an average binding frequency of 9.9 $\pm 1.8 \%$, whereas $\mathrm{gp}^{-} 0^{-}$and $\mathrm{gL}^{-}$virions have binding frequencies of $20.4 \pm 8.3 \%$ and $4.1 \pm 1.4 \%$, respectively (Figure 1B). Strikingly, the removal of gp70 from the viral surface did not decrease virus GAG-binding capacity, but even increased it by a 2 -fold factor. On the contrary, removal of $\mathrm{gL}$ strongly impedes the ability of virions to bind GAGs, whereas $\mathrm{gp} 70^{-} \mathrm{gL}^{-}$virions showed only very little binding $(1.1 \pm 0.5 \%)$, indicating that MuHV-4 virions binding to GAGs is mostly mediated by $\mathrm{gH} / \mathrm{gL}$ and/or gp70. This observation was further confirmed by virus binding assays on living cells using fluorescence microcopy (Figure 1B).

Insight into Glycoprotein-GAG Binding Free-Energy Landscapes. Next, we wanted to extract the kinetic parameters describing the interaction of the individual glycoproteins to GAGs. As the binding force of a biomolecular interaction depends on the rate at which the partners are pulled apart (the loading rate (LR)), ${ }^{15}$ we performed approach and retraction cycles at varying constant tip retraction speeds, ranging from $100 \mathrm{~nm} / \mathrm{s}$ to $20 \mu \mathrm{m} / \mathrm{s}$. The tip velocity is directly proportional to the $\mathrm{LR}[\mathrm{pN} / \mathrm{s}]=v_{\text {tip }}[\mathrm{nm} / \mathrm{s}] \times k_{\text {eff }}[\mathrm{pN} / \mathrm{nm}]$ where $k_{\text {eff }}$ is the effective spring constant of the whole system cantilever-linker-virus-probed surface). As the linker-virussurface system can change its local orientation and as the rupture of interactions can occur at various moments of the tip retraction movement, the stiffness of the system varies for each rupture event measured. This means that for a single tip retraction velocity, a large span of LRs are applied to the virus-GAG interactions. Therefore, applying a few different tip retraction speeds allows obtaining a continuous force distribution spanning over a wide range of LRs. Binding forces and loading rates were extracted from each adhesive curve and displayed in a dynamic force spectroscopy (DFS) plot (Figure 1C). Binding forces were then analyzed as previously described $^{5,13,16}$ (Figure 1 D, E, H and Figure S3). For the gp $70^{-}$virions (LR3 presented in Figure $1 \mathrm{E}$ and other equally important LRs presented in Figure S3), the histograms revealed four peaks, appearing between 90 and $400 \mathrm{pN}$. The relatively high value of the binding forces together with the high binding frequency observed with this virus indicates that multivalency played an important role in binding of gp70virions. This could possibly reduce the chances to establish single glycoprotein-GAG interactions. Indeed, although typical lectin-carbohydrate interactions are usually in the range $40-100 \mathrm{pN}^{13,17}$ this is seldom observed with gp70virions. Therefore, to reduce the effect of multivalency in probing interactions between $\mathrm{gp} 70^{-}$virions and GAGs, we injected free anti-gH/gL antibodies in situ. After blocking, histograms revealed a first peak in the range $50-100 \mathrm{pN}$, which was not observed with gp $70^{-}$virions (Figure $1 \mathrm{~F}$ ). A second peak in the range $100-160 \mathrm{pN}$ was also observed and matched the position of the first peak observed with gp $70^{-}$ virions. We then compared the positions of these peaks with the histograms from the WT virus (Figure 1D and Figure S3) and observed that two first main peaks were present at the same positions as the ones observed with the gp $70^{-}$virions in the presence of anti-gH/gL antibodies. From this, we concluded that the interaction of a single $\mathrm{gH} / \mathrm{gL}$ glycoprotein with GAGs ruptures in the range $50-100 \mathrm{pN}$ (depending on the LR) and that the first peak observed on $\mathrm{gp} 70^{-}$virions histograms arose from the simultaneous rupture of two $\mathrm{gH} /$ gL-GAG interactions. To further validate this conclusion, we also evaluated the stiffness (or effective spring constant) of the complex probed from the slope of the retraction curve before the rupture events (on force vs tip-sample separation curves). We compared the stiffnesses extracted from curves with binding forces in the range 50-95 $\mathrm{pN}$ with the ones from curves showing rupture events in the range $105-145 \mathrm{pN}$ (Figure $1 \mathrm{G}$ ). The mean stiffnesses extracted were significantly higher for the larger interactions suggesting multiple springs in parallel increasing the overall rigidity of the interaction. ${ }^{18}$ Together these results confirm that single-molecule rupture events can only be observed with gp $70^{-}$virions upon injection of an antibody and that multiple parallel bonds between virions and GAGs are already established within a millisecond contact time. Finally, we further fitted the histograms with Gaussian curves at higher forces and found similarly positioned peaks on both histograms from WT and gp $70^{-}$virions. From these fits, we observed that the majority of binding events observed with $\mathrm{WT}$ virions arose from $\mathrm{gH} / \mathrm{gL}-\mathrm{GAG}$ interactions. Smaller peaks also appear on the histograms obtained for WT virions, which could not be correlated with similarly positioned peaks on gp70-deficient virions histograms. However, these peaks are observed for $\mathrm{gL}^{-}$virions (Figure $1 \mathrm{H}$ and Figure S3) and therefore attributed to gp70-GAG interactions (red peaks). 

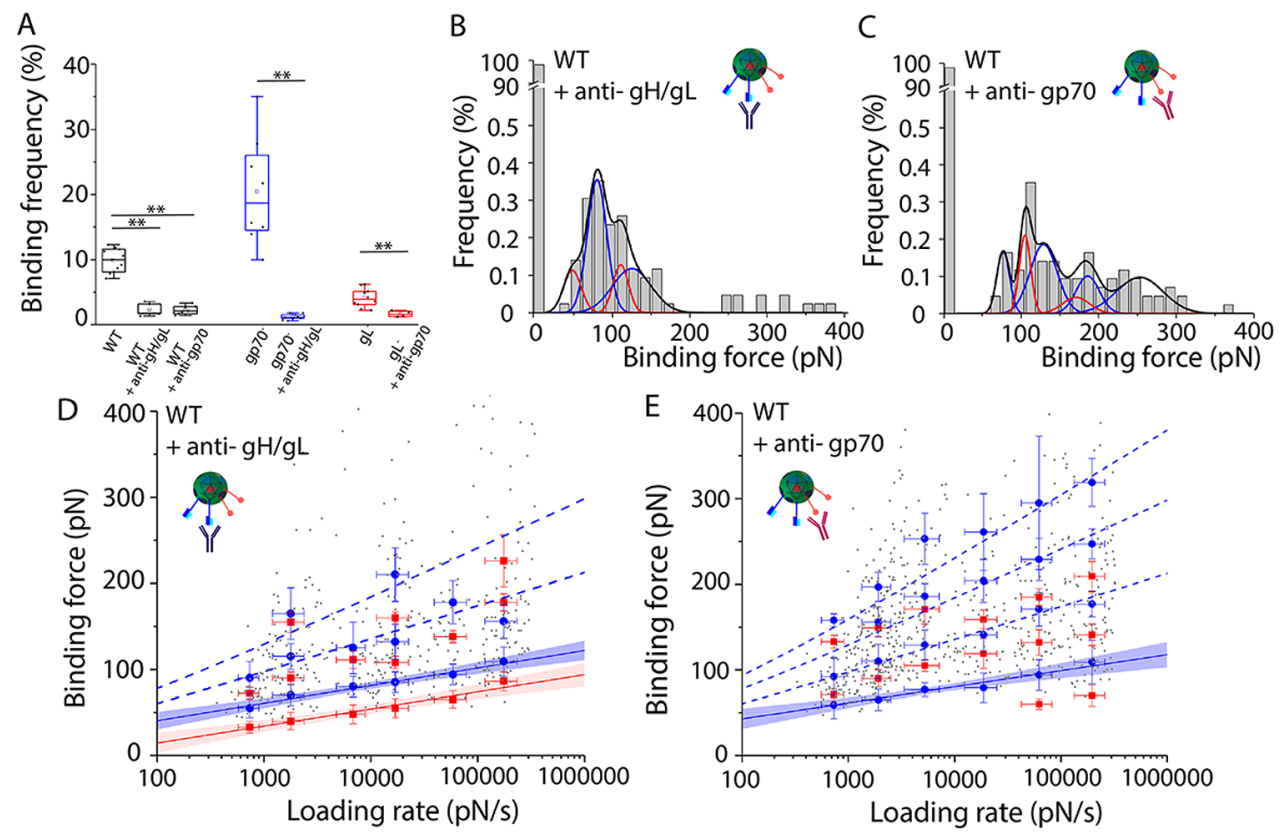

Figure 2. WT MuHV-4 virions binding to GAG-coated surfaces in the presence of antibodies directed against GAG-binding viral glycoproteins. (A) Box plot of binding frequencies of the different viral particles in absence and presence of antibodies directed against viral glycoproteins $\mathrm{gH} / \mathrm{gL}$ or gp70 ( $* *$ indicate $p$-values $<0.05$ on paired sample $t$ tests comparing the binding frequencies of virions before and after antibody injection). (B) Distribution of binding forces of WT virions in the presence of anti-gH/gL antibodies, plotted as histograms for the third LR range (3150-10 000 $\mathrm{pN} / \mathrm{s})(\mathrm{N}=80)$. The histogram is fitted with multigaussian fits. (C) Distribution of binding forces of WT virions in the presence of anti-gp70 antibodies, plotted as histograms for the third LR range. The histogram is fitted with multigaussian fits $(N=85)$. (D) DFS plot reporting the average values of the force distribution for WT MuHV-4-GAG interactions in the presence of anti-gH/gL antibodies $(N=810)$, enabling analysis with the Bell-Evans model (full lines). Dashed lines represent the predicted binding forces for multiple uncorrelated interactions breaking simultaneously, according to the Williams-Evans model. Gray dots represent the force and LR values extracted from each individual FD curve. (E) DFS plot reporting the average values of the force distribution for WT MuHV-4-GAG interactions in the presence of anti-gp70 antibodies $(N=$ 600), enabling analysis with the Bell-Evans model (full lines). Dashed lines represent the predicted binding forces for multiple uncorrelated interactions breaking simultaneously, according to the Williams-Evans model. Gray dots represent the force and LR values extracted from each individual FD curve. Data displayed in B-E were acquired from nine independent experiments.

$\mathrm{gH} / \mathrm{gL}$ Establishes Stable Interaction with GAGs. Using the Bell-Evans model, ${ }^{19,20}$ we next extracted the kinetic parameters describing the interaction between a single viral glycoprotein and GAGs (Figure 1I, full lines). For $\mathrm{gH} / \mathrm{gL}$, the distance to the transition state $\left(\mathrm{x}_{\beta}\right)$ was $0.49 \pm 0.06 \mathrm{~nm}$ and the kinetic off rate $\left(k_{\text {off }}\right)$ equal to $0.09 \pm 0.08 \mathrm{~s}^{-1}$. For gp70, the values were $x_{\beta}=0.61 \pm 0.04 \mathrm{~nm}$ and $k_{\text {off }}=1.05 \pm 0.52 \mathrm{~s}^{-1}$. Using these parameters, we applied the Williams-Evans model $^{21}$ to predict the binding forces of simultaneous breaking multiple uncorrelated interactions (dashed lines). These predictions are in good agreement with the mean binding forces observed and revealing that a vast majority of events involve $\mathrm{gH} / \mathrm{gL}$.

Impeding Glycoprotein-GAG Interactions with Specific Antibodies. After having decipher the attachment mechanism of MuHV-4 virions to GAGs, we wanted to understand how antibodies can impede virions binding. Therefore, we probed these interactions in the presence of anti-gH/gL and anti-gp70 antibodies (Figure S4). The binding frequencies of all virions drastically decreased upon injection of either anti-gH/gL or anti-gp70 antibodies (Figure 2A) suggesting that viral interactions with GAGs is prevented by specific antibodies targeting the viral glycoproteins. This also suggests that steric hindrance induced by the size of antibodies might play a role in the binding frequency decrease, as both the anti-gp70 antibodies, but also anti-gN (a non-GAG-binding viral surface glycoprotein) antibodies (Figure S5) strongly limit the interactions. By looking carefully at the binding force histograms, marked differences are observed on the impact of the two antibodies (Figure 2B, C). In the presence of anti-gH/ $\mathrm{gL}$ antibodies, binding events with rupture forces between 200 and $300 \mathrm{pN}$ are hardly observed, whereas this is not the case with anti-gp70 antibodies, indicating that anti-gH/gL antibodies strongly reduce the multivalency of the interactions while anti-gp70 antibodies do not.

Virions Binding to Living Cells. After having studied virus binding on model substrates, we probed the same interactions in the cellular context. To compare virus-binding properties to cell surface GAGs, we cocultured two Chinese hamster ovary $(\mathrm{CHO})$ cell lines: a cell line expressing GAGs (named $\mathrm{GAG}^{+}$) and carrying the actin-mCherry transgene and a cell line deficient in GAGs expression $\left(\mathrm{GAG}^{-}\right)$. Monolayers of cells were imaged using a combined AFM and epifluorescence microscope under physiologically relevant conditions $\left(37{ }^{\circ} \mathrm{C}, 95 \%\right.$ relative humidity and $5 \% \mathrm{CO}_{2}$ ). Fluorescence microscopy was used to identify the cell lines and select areas in which cells from both cell lines were adjacent (Figure S6). On these areas, AFM tips functionalized with MuHV-4 particles were used to record an AFM topography image together with an adhesion map (Figure 3A, B, E, F, I, J). To evaluate the binding frequencies of virions toward GAGs in the cellular context, we extracted the number of binding events on $\mathrm{GAG}^{+}$cells, with $\mathrm{GAG}^{-}$cells serving as reference. For $\mathrm{GAG}^{-}$cells, the binding frequency remains constant for both WT and mutant MuHV-4 virions and reflects the binding toward specific entry receptors located on the plasma 

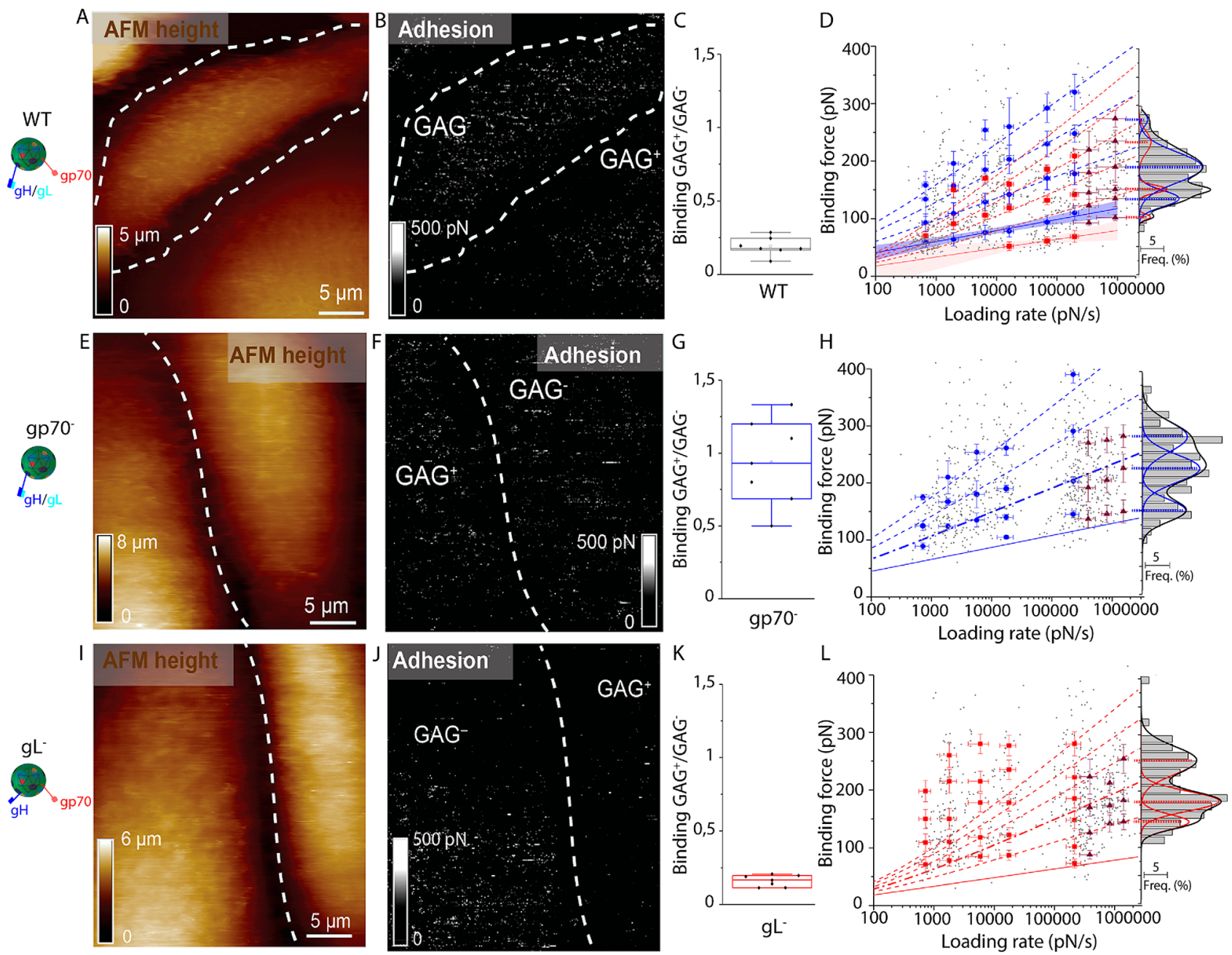

Figure 3. MuHV-4 WT and mutant virions binding to GAGs on cell surfaces. (A) AFM topography image of adjacent GAG ${ }^{+}$and $\mathrm{GAG}^{-}$cells. (B) Corresponding adhesion map, showing interactions between WT MuHV-4 viral particles and imaged cells. Data recorded with WT virions are representative of 32 measurements from nine different experiments. (C) Boxplot of the relative binding frequency $\left(\mathrm{GAG}^{+} / \mathrm{GAG}^{-}\right)$observed for WT virions. (D) DFS plot of WT virion-GAG interactions including data points originating from binding events of virions on cells $(N=340)$. Gray dots represent the force and LR values extracted from each individual FD curve. (E) AFM topography image of adjacent GAG ${ }^{+}$and GAG cells. (F) Corresponding adhesion map, showing interactions between gp70-deficient viral particles and imaged cells. Data recorded with gp $70^{-}$ virions are representative of 18 measurements from 5 different experiments. (G) Boxplot of the relative binding frequency $\left(\mathrm{GAG}^{+} / \mathrm{GAG}^{-}\right.$) observed for gp $70^{-}$virions. (H) DFS plot of gp $70^{-}$virion-GAG interactions including data points originating from binding events of virions on cells $(N=480)$. Gray dots represent the force and LR values extracted from each individual FD curve. (I) AFM topography image of adjacent $\mathrm{GAG}^{+}$and $\mathrm{GAG}^{-}$cells. (J) Corresponding adhesion map, showing interactions between gL-deficient viral particles and imaged cells. Data recorded with $\mathrm{gL}^{-}$virions are representative of 15 measurements from four different experiments. $(\mathrm{K})$ Boxplot of the relative binding frequency $\left(\mathrm{GAG}^{+} /\right.$ $\mathrm{GAG}^{-}$) observed for $\mathrm{gL}^{-}$virions. (L) DFS plot of $\mathrm{gL}^{-}$virion-GAG interactions including data points originating from binding events of virions on cells $(N=280)$. Gray dots represent the force and LR values extracted from each individual FD curve.

membrane and more exposed due to the absence of the thick GAG layer covering the cell surface. Therefore, the binding on the $\mathrm{GAG}^{-}$cells was used as normalization to compare the propensity of the different virions to bind cellular GAGs (Figure 3C, G, K). For WT viral particles, the binding frequency toward $\mathrm{GAG}^{+}$cells was approximately 5-fold lower than on $\mathrm{GAG}^{-}$cells $\left(\mathrm{BF}_{\mathrm{GAG}+} / \mathrm{BF}_{\mathrm{GAG}-}=0.19\right)$, as previously observed. ${ }^{13}$ gp70--virions showed a much higher binding frequency to GAGs than WT virions, as the number of binding events on $\mathrm{GAG}^{+}$cells was almost similar to that on $\mathrm{GAG}^{-}$cells $\left(\mathrm{BF}_{\mathrm{GAG}+} / \mathrm{BF}_{\mathrm{GAG}^{-}}=0.94\right) \cdot \mathrm{gL}^{-}$-virions do nearly not interact with cellular GAGs, as evidenced by their extremely low binding frequency to $\mathrm{GAG}^{+}$cells $\left(\mathrm{BF}_{\mathrm{GAG}^{+}} / \mathrm{BF}_{\mathrm{GAG}^{-}}=0.16\right)$. Control experiments using virions deficient in both glyco- proteins gp70 and gL showed almost no binding to GAGpositive cells (Figure S7)

To ensure that the binding events observed resulted from virus-GAG interactions, F-D curves showing specific adhesion events were extracted, analyzed and displayed on the DFS plots previously obtained on model substrates (Figure 3D, H, L). The binding forces measured on the $\mathrm{GAG}^{+}$cells align very well with those previously extracted on the purified GAGs, confirming that the interactions probed on cells were arising from virus-GAG interactions. In addition, it corroborates that $\mathrm{gH} / \mathrm{gL}$ is the most essential glycoprotein involved in GAG binding during early cell surface attachment.

Finally, we evaluated how antibodies can interfere with virus binding directly on living cells. Surprisingly, injection of both anti-gH/gL and anti-gp70 antibodies led to an increase in 

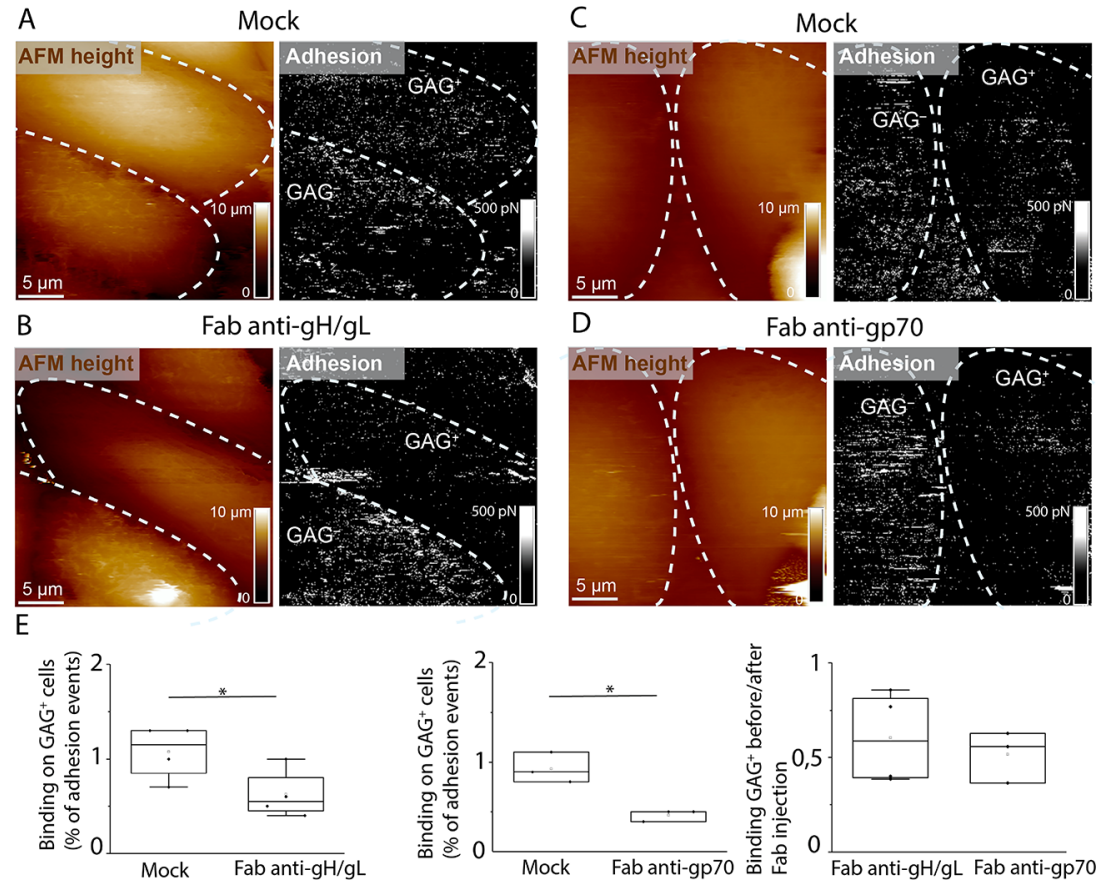

Figure 4. WT MuHV-4 virions binding to GAGs on cell surfaces in the presence of antibodies directed against GAG-binding viral glycoproteins. (A) AFM topography image of adjacent $\mathrm{GAG}^{+}$and $\mathrm{GAG}^{-}$cells and corresponding adhesion map, showing interactions between WT MuHV-4 viral particles and imaged cells. (B) Topography image and adhesion map of the same cells as in A, after injection of anti-gH/gL Fab antibody fragments. (C, D) Topography images and adhesion maps (C) before and (D) after injection of anti-gp70 Fab antibody fragments, recorded with a WT virusbearing AFM tip. (E) Box plots enabling comparison of binding frequencies of virions on GAG-positive cells before and after injection of Fab antibody fragments $(*$ indicates $p$-values $<0.1$ on paired sample $t$ tests comparing the binding frequencies of virions before and after antibody fragment injection).

binding frequencies of virions toward cellular surfaces. We hypothesized that this was due to the free Fc fragments of antibodies that could bind with specific Fc-receptors exposed at the cell surface, ${ }^{22}$ as we observed that after functionalizing AFM tips directly with antibodies, binding events were observed upon probing cellular surfaces (Figure S8). To avoid this, we produced and injected Fab fragments of the antibodies that bind $\mathrm{gH} / \mathrm{gL}$ or gp70 glycoproteins without carrying the $\mathrm{Fc}$ fragments (Figure S9). These anti-gH/gL and anti-gp70 Fab antibodies were validated on GAG model substrates; showing similar properties as the full antibodies (Figure S10). On livings cells, we observed the same tendency, as Fab fragments of anti-gH/gL and anti-gp70 antibodies led to a significant decrease in the binding frequency of virions on $\mathrm{GAG}^{+}$cells (Figure 4). These results indicate that these antibodies impede to a great extent the attachment of virions to GAG exposed on the living cell.

\section{DISCUSSION}

As obligate intracellular parasites, viruses have evolved and adapted to their hosts to efficiently exploit key cellular functions allowing them to complete their viral cycle. ${ }^{23,24}$ Like many other viral species and intracellular pathogens, herpesviruses use GAGs as a first anchor to cellular surfaces. $^{8,25,26}$ In this context, gammaherpesviruses have developed complex mechanisms involving multiple viral glycoproteins to attach to and enter host cells. Two viral surface glycoproteins interacting with GAGs were previously identified for MuHV-4 (gH/gL and gp70), leaving a puzzling redundancy regarding their distinctive properties. We observed the expected GAG-binding capacities of the two glycoproteins: although the deletion of one of them was not sufficient to completely prevent GAG-binding, virions lacking both glycoproteins were unable to bind cellular GAGs. We observed, however, that the capacity to bind GAGs was much more altered for gL-deficient virions than for gp70deficient ones. In addition to that, the measured kinetic off-rate of single $\mathrm{gH} / \mathrm{gL}-\mathrm{GAG}$ interactions was approximately 10 -fold lower than that of single gp70-GAG interactions. This indicates that, in the context of virus early attachment to cell surface GAGs, $\mathrm{gH} / \mathrm{gL}$ provides the major contribution to the binding properties of virions. Viral surface glycoproteins might, however, be constrained to operate sequentially rather than simultaneously, $^{27}$ so that the contribution of gp70 could appear later in the attachment process or even upon virus egress.

In this work, for the first time, we deciphered by AFM the distinct contribution of two presumably redundant viral glycoproteins. By identifying the main viral glycoproteins involved in virus attachment just after its landing on the cell surface, we offer new possibilities to design and strategies to screen antibinding molecule in order to prevent virus infection. Although we showed that full antibody can favor more virus binding to cell surface, Fab antibody shows better capabilities to prevent virus binding. Our results provide a basis for the design and synthesis of antiviral drugs targeting viral glycoproteins to block attachment and entry processes.

\section{ASSOCIATED CONTENT}

\section{Supporting Information}

The Supporting Information is available free of charge at https://pubs.acs.org/doi/10.1021/acs.nanolett.0c04609. 
Detailed methods and Figures S1-S10 (PDF)

\section{AUTHOR INFORMATION}

\section{Corresponding Author}

David Alsteens - Université Catholique de Louvain, Louvain Institute of Biomolecular Science and Technology, Louvain-laNeuve 1348, Belgium; 이 orcid.org/0000-0001-9229-113X; Email: david.alsteens@uclouvain.be

\section{Authors}

Martin Delguste - Université Catholique de Louvain, Louvain Institute of Biomolecular Science and Technology, Louvain-laNeuve 1348, Belgium

Grégoire Le Brun - Université Catholique de Louvain, Louvain Institute of Biomolecular Science and Technology, Louvain-la-Neuve 1348, Belgium

Florian Cotin - Université Catholique de Louvain, Louvain Institute of Biomolecular Science and Technology, Louvain-laNeuve 1348, Belgium

Bénédicte Machiels - University of Liège, ImmunologyVaccinology, FARAH, Liège 4000, Belgium

Laurent Gillet - University of Liège, Immunology-Vaccinology, FARAH, Liège 4000, Belgium

Complete contact information is available at:

https://pubs.acs.org/10.1021/acs.nanolett.0c04609

\section{Author Contributions}

M.D., G.L.B., F.C., B.M., L.G., and D.A. designed experiments. B.M. and L.G. produced all viruses. D.A. set up the AFM chamber. M.D., G.L.B., and F.C. set up and performed AFM experiments. M.D., B.M., and L.G. performed virus binding assays. M.D. produced antibodies and Fab antibody fragments. M.D., G.L.B., F.C., B.M., L.G., and D.A. coanalyzed the experimental data. All authors wrote the paper.

\section{Funding}

This work was supported by the Universite Catholique de Louvain (FSR, Fonds Spéciaux de Recherche) and the Fonds National de la Recherche Scientifique (FRS-FNRS). D.A. and B.M. are Research Associates of the FRS-FNRS and M.D. is a Research Fellow of the FRS-FNRS, respectively. G.L.B. is an FRIA researcher from the FRS-FNRS.

\section{Notes}

The authors declare no competing financial interest.

\section{REFERENCES}

(1) Boulant, S.; Stanifer, M.; Lozach, P.-Y. Dynamics of virusreceptor interactions in virus binding, signaling, and endocytosis. Viruses 2015, 7 (6), 2794-2815.

(2) Gamblin, S.; et al. The structure and receptor binding properties of the 1918 influenza hemagglutinin. Science 2004, 303 (5665), $1838-1842$.

(3) Lindenbach, B. D.; Rice, C. M. The ins and outs of hepatitis C virus entry and assembly. Nat. Rev. Microbiol. 2013, 11 (10), 688700.

(4) Smith, A. E.; Helenius, A. How viruses enter animal cells. Science 2004, 304 (5668), 237-242.

(5) Alsteens, D.; et al. Nanomechanical mapping of first binding steps of a virus to animal cells. Nat. Nanotechnol. 2017, 12 (2), 177. (6) Kukura, P.; et al. High-speed nanoscopic tracking of the position and orientation of a single virus. Nat. Methods 2009, 6 (12), 923.

(7) Yang, J.; Petitjean, S. J. L.; Koehler, M.; Zhang, Q.; Dumitru, A. C.; Chen, W.; Derclaye, S.; Vincent, S. P.; Soumillion, P.; Alsteens, D.; et al. Molecular interaction and inhibition of SARS-CoV-2 binding to the ACE2 receptor. Nat. Commun. 2020, 11, 4541.
(8) Shukla, D.; Spear, P. G. Herpesviruses and heparan sulfate: an intimate relationship in aid of viral entry. J. Clin. Invest. 2001, 108 (4), 503-510.

(9) François, S.; et al. Comparative study of murid gammaherpesvirus 4 infection in mice and in a natural host, bank voles. J. Gen. Virol. 2010, 91 (10), 2553-2563.

(10) De Lima, B. D.; May, J. S.; Stevenson, P. G. Murine gammaherpesvirus 68 lacking gp150 shows defective virion release but establishes normal latency in vivo. J. Virol. 2004, 78 (10), 5103-5112.

(11) Gillet, L.; Adler, H.; Stevenson, P. G. Glycosaminoglycan interactions in murine gammaherpesvirus-68 infection. PLoS One 2007, 2 (4), No. e347.

(12) Gillet, L.; Colaco, S.; Stevenson, P. G. The murid herpesvirus-4 $\mathrm{gH} / \mathrm{gL}$ binds to glycosaminoglycans. PLoS One 2008, 3 (2), No. e1669.

(13) Delguste, M.; et al. Multivalent binding of herpesvirus to living cells is tightly regulated during infection. Sci. Adv. 2018, 4 (8), No. eaat 1273

(14) Delguste, M.; et al. Regulatory mechanisms of the mucin-like region on herpes simplex virus during cellular attachment. ACS Chem. Biol. 2019, 14 (3), 534-542.

(15) Merkel, R.; et al. Energy landscapes of receptor-ligand bonds explored with dynamic force spectroscopy. Nature 1999, 397 (6714), $50-53$.

(16) Newton, R.; et al. Combining confocal and atomic force microscopy to quantify single-virus binding to mammalian cell surfaces. Nat. Protoc. 2017, 12 (11), 2275.

(17) Touhami, A.; et al. Probing specific lectin-carbohydrate interactions using atomic force microscopy imaging and force measurements. Langmuir 2003, 19 (5), 1745-1751.

(18) Rankl, C.; et al. Multiple receptors involved in human rhinovirus attachment to live cells. Proc. Natl. Acad. Sci. U. S. A 2008, 105 (46), 17778-17783.

(19) Evans, E.; Ritchie, K. Dynamic strength of molecular adhesion bonds. Biophys. J. 1997, 72 (4), 1541-1555.

(20) Evans, E. A.; Calderwood, D. A. Forces and bond dynamics in cell adhesion. Science 2007, 316 (5828), 1148-1153.

(21) Evans, E.; Williams, P., Dynamic force spectroscopy. In Physics of Biomolecules and Cells; Flyvbjerg, F., Jülicher, F., Ormos, P., David, F., Eds.; Springer: Berlin, 2002; p 145-204.

(22) Rosa, G. T.; et al. IgG fc receptors provide an alternative infection route for murine gamma-herpesvirus-68. PLoS One 2007, 2 (6), No. e560.

(23) Pelkmans, L.; Helenius, A. Insider information: what viruses tell us about endocytosis. Curr. Opin. Cell Biol. 2003, 15 (4), 414-422.

(24) Borza, C. M.; Hutt-Fletcher, L. M. Alternate replication in B cells and epithelial cells switches tropism of Epstein-Barr virus. Nat Med. 2002, 8 (6), 594-599.

(25) Spillmann, D. Heparan sulfate: anchor for viral intruders? Biochimie 2001, 83 (8), 811-817.

(26) Wadstrom, T.; Ljungh, A. Glycosaminoglycan-binding microbial proteins in tissue adhesion and invasion: key events in microbial pathogenicity. J. Med. Microbiol. 1999, 48 (3), 223-233.

(27) Gillet, L.; Stevenson, P. G. Evidence for a multiprotein gamma2 herpesvirus entry complex. J. Virol. 2007, 81 (23), 13082-13091.

\section{NOTE ADDED AFTER ASAP PUBLICATION}

Due to a production error, this paper was published ASAP on December 29, 2020, with errors in Figure 1. The corrected version was reposted on December 30, 2020. 